\title{
Combined laser and atomic force microscope lithography on aluminum: Mask
} fabrication for nanoelectromechanical systems

\author{
Berini, Abadal Gabriel; Boisen, Anja; Davis, Zachary James; Hansen, Ole; Grey, Francois
}

Published in:

Applied Physics Letters

Link to article, DOI:

10.1063/1.124106

Publication date:

1999

Document Version

Publisher's PDF, also known as Version of record

Link back to DTU Orbit

Citation (APA):

Berini, A. G., Boisen, A., Davis, Z. J., Hansen, O., \& Grey, F. (1999). Combined laser and atomic force microscope lithography on aluminum: Mask fabrication for nanoelectromechanical systems. Applied Physics Letters, 74(21), 3206-3208. https://doi.org/10.1063/1.124106

\section{General rights}

Copyright and moral rights for the publications made accessible in the public portal are retained by the authors and/or other copyright owners and it is a condition of accessing publications that users recognise and abide by the legal requirements associated with these rights.

- Users may download and print one copy of any publication from the public portal for the purpose of private study or research.

- You may not further distribute the material or use it for any profit-making activity or commercial gain

- You may freely distribute the URL identifying the publication in the public portal 


\title{
Combined laser and atomic force microscope lithography on aluminum: Mask fabrication for nanoelectromechanical systems
}

\author{
G. Abadal, ${ }^{\text {a) }}$ A. Boisen, Z. J. Davis, O. Hansen, and F. Grey \\ Mikroelektronik Centret, Technical University of Denmark, Bldg. 345e, DK-2800 Lyngby, Denmark
}

(Received 29 January 1999; accepted for publication 29 March 1999)

\begin{abstract}
A direct-write laser system and an atomic force microscope (AFM) are combined to modify thin layers of aluminum on an oxidized silicon substrate, in order to fabricate conducting and robust etch masks with submicron features. These masks are very well suited for the production of nanoelectromechanical systems (NEMS) by reactive ion etching. In particular, the laser-modified areas can be subsequently locally oxidized by AFM and the oxidized regions can be selectively removed by chemical etching. This provides a straightforward means to define the overall conducting structure of a device by laser writing, and to perform submicron modifications by AFM oxidation. The mask fabrication for a nanoscale suspended resonator bridge is used to illustrate the advantages of this combined technique for NEMS. (c) 1999 American Institute of Physics. [S0003-6951(99)00221-1]
\end{abstract}

The need for miniaturization of microelectromechanical systems is increasing, leading to a range of devices with a functionality that depends on submicron definition of key features. One of the challenges for this emerging field of nanoelectromechanical systems (NEMS) is to fabricate suspended beams with widths in the $100 \mathrm{~nm}$ range for use in high-frequency resonators and Fabry-Perot interferometers. ${ }^{1,2}$ For the fabrication of these devices, advanced nanolithographic processes are needed. Most of the nanomechanical devices realized to date have been fabricated using e-beam pattern definition. ${ }^{2}$ Lithography by atomic force microscopy (AFM) and scanning tunneling microscopy (STM) has also been applied, 3,4 although these techniques are usually not sufficiently fast or robust for pattern definition of a complete device.

We have recently demonstrated ${ }^{5}$ that patterning of an aluminum film by a direct-write laser system or an AFM provides a means of forming positive as well as negative etch masks with an extremely high selectivity to fluorinebased reactive ion etch (RIE) processes. This high selectivity makes it possible to fabricate high-resolution suspended structures. In this letter, we describe for the first time a combination of the laser and AFM writing on Al for rapid patterning of a mask for a complete NEMS device. The combination is based on AFM postprocessing of aluminum regions which have already been modified by laser writing. To illustrate this approach, we fabricate the etch mask for a resonator, where the main features of the device are patterned by laser writing, and subsequently an AFM is used to define a resonator bridge with submicron dimensions. The conductivity of the laser-modified aluminum film is also investigated and we find that it can be used as an electrical contact or a conductor in the fabricated structures. This facilitates the combination of advanced nanomechanical structures with integrated microelectronics, which is particularly significant

${ }^{a)}$ Current affiliation: Department d'Enginyeria Electrònica, Universitat Autònoma de Barcelona. 08193-Bellaterra.

Electronic mail: gabadal@cc.uab.es since aluminum-based masks and RIE processes are fully compatible with standard complementary metal-oxide semiconductor (CMOS) microelectronics fabrication.

The substrates for patterning are prepared as follows. A thermal oxide layer $250 \mathrm{~nm}$ thick is grown on a $\mathrm{Si}(100)$ substrate and subsequently a 5-7-nm-thick Al film is e-beam evaporated. Laser patterning of the Al layer is done in air by locally exposing the sample to a continuous argon laser beam $(\lambda=488 \mathrm{~nm}) .^{6}$ The beam is focused and laterally positioned on the sample surface by means of an optical setup and a computer-controlled lateral translation system that allows writing of complex two-dimensional structures. This direct laser writing technique is a thermal process that induces a reaction between the $\mathrm{Al}$ thin film and the silicon oxide from the substrate. ${ }^{5}$ Secondary ion mass spectrometry measurements and selective etching experiments indicate that the laser-annealed mask is an $\mathrm{Al} / \mathrm{Si}$ alloy with a thin (1-2 nm) top layer of aluminum oxide. Below a critical laser power, at which the Al evaporates, the linewidth decreases for decreasing laser power and increasing writing speed. The smallest line width achieved with this technique is $500 \mathrm{~nm}$.

Once the sample has been patterned by laser annealing, the exposed zones withstand an $85 \%$ phosphoric acid etch at room temperature, whereas the unpatterned $\mathrm{Al}$ is etched. The laser-written structures can then be used as masks for fluorine-based reactive ion etching (RIE) in order to fabricate suspended mechanical structures on the sample surface. ${ }^{5}$

In order to characterize the electrical conductivity of the laser-exposed $\mathrm{Al}$, a set of eight test structures has been written for six different laser powers with a laser spot diameter of approximately $0.5 \mu \mathrm{m}$ and a writing speed of $1 \mathrm{~mm} / \mathrm{s}$. A test structure consists of two $200 \times 200 \mu \mathrm{m}^{2}$ pads connected by a $200-\mu \mathrm{m}$-long wire with a width ranging from 0.5 to 10 $\mu \mathrm{m}$. Based on the current-voltage $(I-V)$ characteristics for the test structures, the sheet resistance of the annealed Al can be estimated for each laser power.

The linearity of the $I-V$ characteristics measured on the laser annealed $\mathrm{Al}$ wires [Fig. 1(a)] indicates that an ohmic contact is provided between the external electrical probes, 

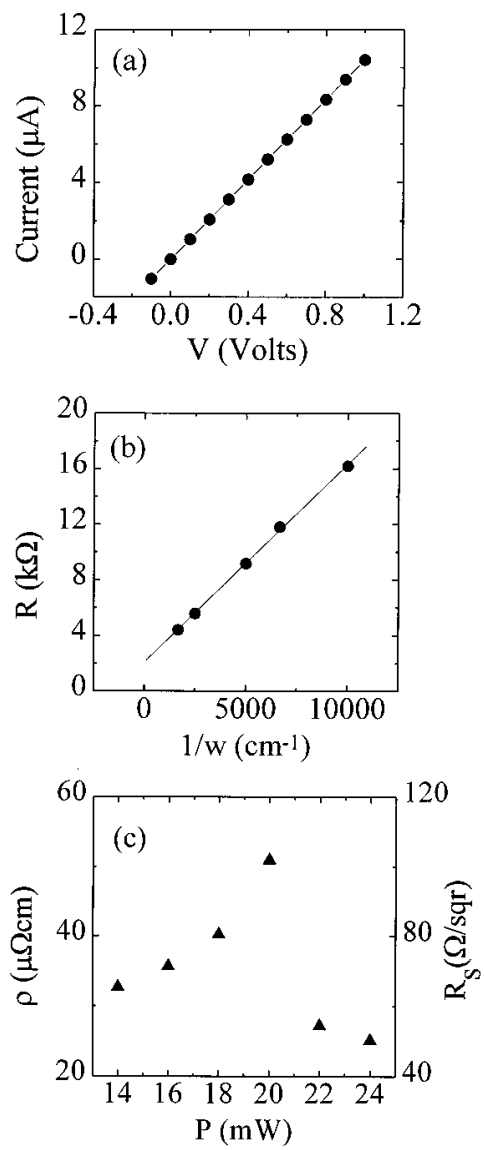

FIG. 1. (a) Current-voltage characteristic measured on a $200 \times 1 \mu \mathrm{m}^{2}$ laser annealed $\mathrm{Al}$ wire. The wire is contacted to external electrical probes through two $200 \times 200 \mu \mathrm{m}^{2}$ contact pads. (b) Electrical resistance of the wires as a function of the length/width ratio. (c) Measured sheet resistance of the wires as a function of the laser power used for writing.

the pads made of the modified $\mathrm{Al}$ layer, and a narrow wire of the same modified Al. The resistance, $R$, obtained from the slope of the $I-V$ characteristics also shows a linear dependence on $l / w$ [Fig. 1(b)], where $l=200 \mu \mathrm{m}$ is the fixed wire length and $w$ is the variable wire width. From the relation between resistance, resistivity $(\rho)$, and geometrical parameters a linear relationship is expected:

$$
R=R_{c, p}+\frac{\rho}{t} \times \frac{l}{w} .
$$

The term $R_{c, p}$ includes the contact and the pad resistance, and $t$ is the annealed Al layer thickness. The slope of the linear relation between $R$ and $l / w$ can be identified as the sheet resistance, $R_{S}=\rho / t$, of the laser-annealed $\mathrm{Al}$ layer. The curve in Fig. 1(c) has been obtained by repeating the sheet resistance measurements for a set of wires written with different laser powers. Assuming that the thickness of the $\mathrm{Al}$ film, $t=5 \mathrm{~nm}$, is not altered by the laser annealing process, we deduce values between 25 and $50 \mu \Omega \mathrm{cm}$ for the annealed $\mathrm{Al}$ resistivity. This is one order of magnitude larger than the resistivity of pure $\mathrm{Al}(2.65 \mu \Omega \mathrm{cm})$. The existence of a maximum sheet resistance for a laser power of $20 \mathrm{~mW}$ might suggest a phase change of the annealed Al.

A thin Al layer can also be patterned by AFM-based lithography. The microscope is operated in contact mode and the AFM probe is biased at a negative voltage with respect to the sample. As a result the $\mathrm{Al}$ film is locally oxidized by an Downloaded 24 Jan 2010 to 192.38.67.112. Redistribution subject anodization mechanism. ${ }^{7}$ A commercial AFM has been adapted to perform the experiments in a humidity-controlled ambient. Silicon-nitride AFM probes have been coated with a 15-nm-thick Ti film in order to make them conductive. Line widths of $100 \mathrm{~nm}$ are reproducibly obtained using negative tip voltages in the range of -10 to $-20 \mathrm{~V}$, scan speeds below $10 \mu \mathrm{m} / \mathrm{s}$, and relative humidities around $40 \%$.

The AFM written regions can, like the laser written regions, withstand an $\mathrm{Al}$ etch and the resulting mask can be used directly for RIE of nm-sized mechanical structures. Moreover, we have discovered that the AFM can be used to locally further oxidize a laser-annealed $\mathrm{Al}$ mask. This gives a way to combine laser and AFM writing in which small parts of the laser written pattern can be electrically insulated or removed. For combined laser and AFM writing the laser annealed mask needs to be located by AFM. In order to achieve good topographical contrast the Al, which has not been exposed by laser, is removed before local AFM oxidation. The laser-annealed $\mathrm{Al}$ regions oxidized by AFM can be removed in a standard $\mathrm{Al}_{2} \mathrm{O}_{3}$ etch solution (85\% phosphoric acid, deionized water, and chromium trioxide) at $50^{\circ} \mathrm{C}$.

To demonstrate the possibility of locating and patterning a laser-defined region by AFM, we have modified a 2- $\mu \mathrm{m}-$ wide laser-defined $\mathrm{Al}$ wire. The wire is easily found by AFM and at the center of the wire a $5 \times 5 \mu \mathrm{m}^{2}$ region has been scanned at $8 \mu \mathrm{m} / \mathrm{s}$ and with a tip bias of $-20 \mathrm{~V}$ with respect to the sample. The scanned zone of the wire is seen as a protrusion in a subsequent AFM image, and after rinsing the sample in an $\mathrm{Al}_{2} \mathrm{O}_{3}$ etch solution, the zone is seen as a depression with a depth corresponding to the thickness of the Al layer. Furthermore, the wire conductivity becomes zero after scanning the central wire region. These observations demonstrate that the total thickness of the laser-annealed $\mathrm{Al}$ layer can be oxidized by AFM. The wire conductivity has also been reduced in a controlled way by oxidizing the edges of the $\mathrm{Al}$ wire by AFM, in a manner similar to Snow et al. ${ }^{8}$

The possibility of oxidizing the laser-annealed Al layer by AFM allows us to combine the two writing techniques in order to take advantage of the speed of direct laser writing and the nanometer resolution of the AFM lithography. As an example of the possibilities of the combined AFM and laser writing, we show in Fig. 2(a) sequence of AFM images which illustrates the definition process of an annealed $\mathrm{Al}$ mask for the fabrication of an electrostatically driven nanobridge resonator. First, a mask consisting of three $200 \times 200$ $\mu \mathrm{m}^{2}$ pads connected to three ends of a " $\mathrm{T}$ " shaped structure is defined by laser writing. As shown in Fig. 2(a), the topographical contrast achieved after $\mathrm{Al}$ etching allows us to image the structure by AFM and to position the tip very precisely on any part of the T structure. Thus, it is possible to modify the laser-annealed area with high accuracy. In Fig. 2(b) two regions have been oxidized on the $\mathrm{T}$ structure. The oxidized regions are seen as protrusions in the AFM image. The oxidation was performed at tip-sample bias of $-20 \mathrm{~V}$ and at a scan speed of $5 \mu \mathrm{m} / \mathrm{s}$. By removing the oxidized $\mathrm{Al}$ in the $\mathrm{Al}_{2} \mathrm{O}_{3}$ etch [Fig. 2(c)], the width of the upper part of the $\mathrm{T}$ structure is reduced from $2 \mu \mathrm{m}$ to $400 \mathrm{~nm}$. Moreover, the upper part is separated by $200 \mathrm{~nm}$ from the lower part. The final result is a laser-annealed $\mathrm{Al}$ mask consisting of a to AIP license or copyright; see http://apl.aip.org/apl/copyright.jsp 

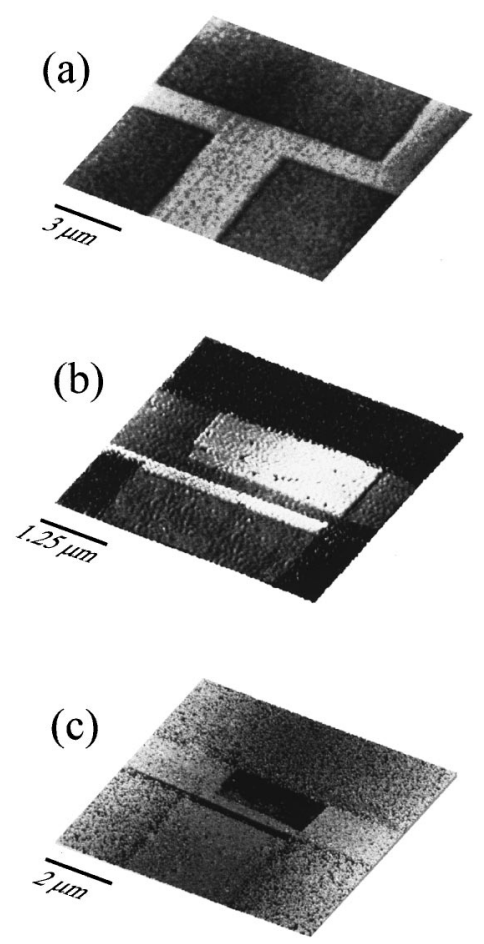

FIG. 2. AFM images (contact mode) illustrating the definition process of an annealed $\mathrm{Al}$ mask used to fabricate a nanometer scale bridge resonator. (a) The $\mathrm{T}$ shaped zone of the laser defined mask after Al etching. (b) The same structure after local oxidation by AFM lithography. (c) Central part of the final mask after etching of the oxidized regions.

very narrow line connected to two pads and a larger strip connected to a third pad.

In conclusion, we have described a simple, flexible, and resistless mask fabrication technique for the definition of na- noelectromechanical devices. AFM lithography has been used to locally oxidize laser-defined $\mathrm{Al}$ masks, and the oxidized regions have been selectively removed. It is thus possible to electrically insulate or physically reduce the size of regions of the laser defined mask. The combination of AFM and laser writing is advantageous because it is much faster than using AFM alone and it reduces wear of the AFM tip, while still ensuring mechanical and electrical integrity of the fine AFM-written structures and the larger-scale laser written patterns. The laser-annealed $\mathrm{Al}$ mask is an $\mathrm{Al} / \mathrm{Si}$ alloy which is seen to exhibit an ohmic behavior with a resistivity comparable to the resistivity of pure Al. Thus, it is possible to use the laser-modified $\mathrm{Al}$ film as an etch mask as well as an electrical contact or conductor in nanoelectromechanical devices. The processes described here are CMOS compatible, which makes them promising tools for the integration of nanoelectromechanical devices with standard microelectronics.

This work was partially funded by the TMR-project NANOFAB.

${ }^{1}$ A. N. Cleland and M. L. Roukes, Appl. Phys. Lett. 69, 2653 (1996).

${ }^{2}$ D. W. Carr and H. G. Craighead, J. Vac. Sci. Technol. B 15, 2760 (1997).

${ }^{3}$ E. S. Snow, P. M. Campbell, and P. J. McMarr, Nanotechnology 7, 434 (1996).

${ }^{4}$ H. Hamanaka, T. Ono, and M. Esashi, Proceedings IEEE, The Tenth Annual International Workshop on Micro Electro Mechanical Systems, 1997 (unpublished), pp. 153-158.

${ }^{5}$ A. Boisen, K. Birkelund, O. Hansen, and F. Grey, J. Vac. Sci. Technol. B 9, 2977 (1998).

${ }^{6}$ M. Müllenborn, H. Dirac, and J. W. Petersen, Appl. Phys. Lett. 66, 3001 (1995).

${ }^{7}$ M. Ishii and K. Matsumoto, Jpn. J. Appl. Phys., Part 1 Part 1 34, 1329 (1995).

${ }^{8}$ E. S. Snow, D. Park, and P. M. Campbell, Appl. Phys. Lett. 69, 3918 (1996). 